\title{
Cognition in Restless Legs Syndrome
}

\author{
Ki-Young Jung \\ Department of Neurology, Seoul National University Hospital, Seoul National University College of Medicine, Seoul, Korea
}

Received June 15, 2015

Revised June 22, 2015

Accepted June 22, 2015

Address for correspondence

Ki-Young Jung, MD, PhD

Department of Neurology,

Seoul National University

Hospital, Seoul National

University College of Medicine,

101 Daehak-ro, Jongno-gu,

Seoul 110-744, Korea

Tel: $+82-2-2072-6649$

Fax: +82-2-2072-2474

E-mail: jungky@snu.ac.kr
Cognition is the mental abilities related to knowledge which includes attention, memory, language, judgment, and decision making. Cognitive function can be affected by several factors including attention, emotion, and sleep. In restless legs syndrome (RLS), cognitive dysfunction may ensue due to sleep disturbance, emotional disorders, and attention deficit which are common in RLS. However, it remains unclear whether cognitive performance is decreased in patients with RLS. To date, there are ten published articles which studied cognition in RLS. Five studies reported cognitive dysfunction of RLS, particularly decreased performance in the attention, frontal executive function, and mental flexibility function. Three reported no significant differences in cognitive performance between RLS patients and control subjects. Two studies reported even better performance in patients with RLS compared with control subjects. Dopamine agonist may improve cognitive performance in RLS. However, it remains unclear whether this is direct effect on RLS symptoms or indirect effect including improved sleep duration, daytime sleepiness and depression, or both. Event-related potential studies demonstrated reduced P300 amplitude either oddball task or Sternberg working memory task. Larger sample and studies using functional neuroimaging study are needed to confirm whether cognition is impaired or not in RLS.

J Sleep Med 2015;12(1):1-6

Key Words: Restless legs syndrome, Cognition, Working memory, Event-related potential, P300.

\section{Introduction}

Cognition is the set of all mental abilities and processes related to knowledge, which encompasses attention, memory, judgment and evaluation, reasoning and computation, problem solving and decision making as well as comprehension and production of language. Cognitive function can be affected by several factors including emotion and sleep. Restless legs syndrome (RLS) is a sensorimotor network disorder characterized by an irresistible urge to move the legs during rest accompanied by unpleasant and disturbing sensations in the legs. ${ }^{1}$ In RLS, cognitive dysfunction may ensue due to sleep disturbance, emotional disorders, and attention deficit which are common in RLS. There are a few unresolved issues regarding cognition in RLS. The first question should be "Is cognition really impaired in RLS?”. Although recent studies have reported that moderate to severe RLS patients may have significant cognitive deficits, primarily in executive functioning, compared with healthy control subjects, ${ }^{2-5}$ other studies found no difference in cognitive function between RLS pa-

This is an Open Access article distributed under the terms of the Creative Commons Attribution Non-Commercial License (http://creativecommons.org/licenses/by-nc/3.0) which permits unrestricted non-commercial use, distribution, and reproduction in any medium, provided the original work is properly cited. tients and controls. Therefore, this controversy has been mentioned at the first part of this article. The next question should be "Which cognitive domain(s) are affected in those studies which revealed cognitive dysfunction in RLS?". After these discussion, factors associated with cognitive dysfunction in RLS should be mentioned. Lastly, the effect of dopaminergic agent on cognitive dysfunction in RLS needs to be addressed. In this review, the answers for these questions will be explored. At the end of this review, sophisticated measures which might help identifying cognitive functioning in RLS such as eventrelated potential (ERP) studies will be mentioned.

\section{Is Cognitive Function in RLS Really Impaired?}

To date, there are ten published articles which studied cognition in RLS (Table 1). Five were community-based studies and the rest were hospital-based. ${ }^{3,5-8}$ Five studies reported cognitive dysfunction in RLS patients. ${ }^{2-5,7}$ Three reported no significant differences in cognitive performance between RLS patients and control subjects. ${ }^{6,89}$ In these three studies, RLS patients were older and had less severe RLS in contrast to the five studies which reported cognitive dysfunction. Surprisingly, the other two studies reported that even better cognitive 
function was observed in patients with RLS compared to control subjects. ${ }^{10,11}$

The discrepancies among studies may be attributable to several factors such as differences in study samples, use of different age groups (middle-aged vs. old-aged), types of neuropsychological tests administered (full batteries vs. limited to frontal function tests), and medication effects (unmedicated vs. medicated).

\section{Which Cognitive Domains Are Affected in RLS?}

Among the five studies which reported cognitive dysfunction, only one study performed comprehensive neurocognitive tests (Table 2). ${ }^{7}$ The other four studies included limited tests, focusing on only the frontal executive and mental flexibility tests. ${ }^{2-5}$ All of the five studies demonstrated decreased performance in attention, frontal executive function, and mental flexibility function. In contrast, only one study revealed dysfunction in verbal and visuospatial memory, working memory, and language functions unlike the other studies, which revealed normal cognitive function in these areas. ${ }^{7}$

\section{Potential Factors Associated with Cognitive Dysfunction in RLS}

Legs discomfort, which is aggravated by lying down to sleep in the evening, hinders beginning and maintaining of sleep in severe RLS patients. This leads RLS patients to suffer from sleep disturbance, causing chronic partial sleep loss. ${ }^{1,12}$ Since cognitive function is affected by sleep loss, sleep deprivation due to RLS symptoms might be the cause of cognitive dysfunction in RLS patients.

Another possible mechanism of cognitive dysfunction can be explained by decreased attention due to RLS symptoms itself. Clinically, RLS patients frequently experience hyperactivity and inattention, which can mimic symptoms of attention deficit/hyperactivity disorder (ADHD). Furthermore, RLS might be associated with ADHD. ${ }^{13,14}$

Comorbid psychiatric conditions such as depression and anxiety are common in patients with RLS. ${ }^{15,16}$ Epidemiological studies report a 2- to 4-fold risk of a depressive disorder in patients with RLS compared to healthy controls. As depression is well known to compromise cognitive function, depression might be considered as a contributing factor to the cognitive deficit in patients with RLS. Furthermore, RLS-related sleep loss might cause depressive symptoms, and vice versa. ${ }^{15}$ Thus,

Table 1. Summary of studies which investigated cognition in patients with RLS

\begin{tabular}{|c|c|c|c|c|c|c|}
\hline $\begin{array}{c}\text { Cognitive function in RLS } \\
\text { patients compared to controls }\end{array}$ & $\begin{array}{l}\text { No. of } \\
\text { articles }\end{array}$ & $\begin{array}{c}\text { Total No. of } \\
\text { patients studied (n) }\end{array}$ & $\begin{array}{c}\text { Total No. of } \\
\text { controls studied (n) }\end{array}$ & $\begin{array}{l}\text { Pooled mean age of } \\
\text { patients (years) }\end{array}$ & $\begin{array}{l}\text { Pooled mean age of } \\
\text { controls (years) }\end{array}$ & IRLS \\
\hline Dysfunction $^{2-5,7}$ & 5 & 273 & 680 & 57.2 & 55.9 & 22.3 \\
\hline No change $e^{6,8,9}$ & 3 & 466 & 1898 & 76.0 & 76.3 & 14.9 \\
\hline Better function ${ }^{10,11}$ & 2 & 31 & 30 & 52.5 & 55.3 & 27.0 \\
\hline
\end{tabular}

IRLS: International RLS Severity Scale, RLS: restless legs syndrome

Table 2. Summary of neurocognitive tests from studies reporting cognitive dysfunction in RLS

\begin{tabular}{|c|c|c|c|c|c|}
\hline & \multicolumn{5}{|c|}{ Authors } \\
\hline & Pearson et al. $^{3}$ & Fulda et al. ${ }^{1}$ & Celle et al. ${ }^{4}$ & Fulda et al. $^{2}$ & Galbiati et al. $^{6}$ \\
\hline No. of subjects & 16 & 23 & 173 & 41 & 20 \\
\hline IRLS & NA & 23.5 & 17.1 & NA & 26.2 \\
\hline Attention & NA & Decreased & NA & NA & Decreased \\
\hline Frontal executive & $\begin{array}{l}\text { Decrease in TMT-B, } \\
\text { verbal fluency }\end{array}$ & $\begin{array}{l}\text { Decrease in verbal } \\
\text { fluency }\end{array}$ & $\begin{array}{l}\text { Decrease in verbal } \\
\text { fluency }\end{array}$ & NA & $\begin{array}{l}\text { Decrease in TMT-A, } \\
\text { verbal fluency }\end{array}$ \\
\hline Mental flexibility & NA & Decrease in Stroop & Decrease in Stroop & WCST & $\begin{array}{c}\text { Decrease in Stroop, } \\
\text { WCST }\end{array}$ \\
\hline Working memory & NA & NA & NA & NA & Decreased \\
\hline Verbal memory & NA & NA & NA & NA & Decreased \\
\hline Visuospatial & NA & NA & NA & NA & Decreased \\
\hline Language & NA & NA & NA & NA & Decreased \\
\hline
\end{tabular}

IRLS: International RLS Severity Scale, TMT: trail making test, WCST: Wisconsin card sorting test, NA: not available, RLS: restless legs syndrome 
these factors may affect cognitive function in patients with RLS. Therefore, these factors should be taken into consideration in the evaluation of cognitive dysfunction in RLS (Fig. 1).

Pearson et al. reported that RLS patients show cognitive deficits, particularly in prefrontal function, which are comparable to the loss of a night's sleep. ${ }^{3}$ In a subsequent comparative study of RLS patients and sleep restricted controls, however, the same group found that RLS patients performed significantly better on prefrontal function tests than controls. ${ }^{10}$ Although the authors proposed that RLS subjects might adapt to sleep loss to some extent, sleep loss alone does not appear to explain cognitive dysfunction in RLS.

Fulda et al. showed that RLS patients performed worse than controls in the area of attention and verbal fluency. ${ }^{2}$ The International RLS Severity Scale scores were significantly associated with performance in the cancellation task. However, task performance did not correlated with subjective sleep quality and duration. A community based study reported by Celle et al. revealed that RLS patients had lower cognitive performances at Stroop and Verbal fluency tests than controls

Table 3. Factors associated with cognitive dysfunction in RLS

\begin{tabular}{lll}
\hline & Associated & Not associated \\
\hline Chronic sleep loss & NA & Gamaldo et al. ${ }^{9}$ \\
& & Fulda et al. ${ }^{1}$ \\
& & Celle et al. ${ }^{4}$ \\
RLS severity & Fulda et al. ${ }^{2}$ & Celle et al. ${ }^{4}$ \\
Depression & NA & Fulda et al. ${ }^{1}$ \\
\hline
\end{tabular}

RLS: restless legs syndrome, NA: not available

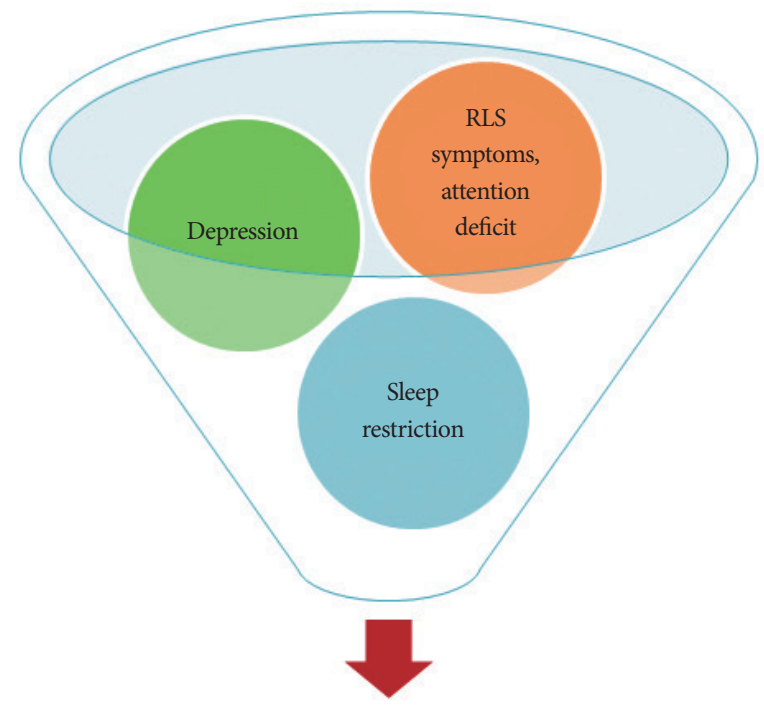

Cognitive dysfunction in RLS

Figure 1. Potential factors affecting cognition in restless legs syndrome (RLS).
(Table 3). ${ }^{4}$ Correlation analysis showed that neither RLS severity score nor degree of sleep disturbances were associated with the degree of cognitive dysfunction, indicating independent effect of RLS on such cognitive tasks. Fulda et al. demonstrated no statistically significant differences in any of the neuropsychological parameters between RLS patients and controls. ${ }^{2}$ However, comparing participants with frequent RLS symptoms to matched controls revealed decreased performance on the Wisconsin Card Sorting Test in RLS patients, suggesting association between cognitive dysfunction and RLS severity. Moon et al. revealed that RLS patients performed better than controls on tests of verbal memory and category word fluency. ${ }^{10}$ RLS patients scored higher Beck depression inventory than controls. To identify whether depression had an impact on cognitive function, we compared cognitive function between patients with moderate to severe depression and those without depression. However, there was no significant differences in cognitive function between patients with moderate to severe depression and those without depression.

\section{Effect of Dopaminergic Treatment on Cognition in RLS}

Dopamine agonists are recommended as a first-line treatment in cases of moderate to severe RLS, improving primary RLS symptoms. ${ }^{17}$ In addition, dopamine agonists improve periodic leg movements during sleep, ${ }^{18}$ depressive symptoms, ${ }^{19}$ and quality of life. ${ }^{20}$ Two studies reported the effects of dopamine agonists on cognition in RLS subjects. Kim et al. studied sixteen drug-naïve RLS patients who were treated with pramipexole for 3 months. ${ }^{21}$ After treatment with pramipexole, severity of RLS symptoms decreased significantly. Sleep quality and depression also improved significantly following treatment. Neuropsychological tests showed significantly improved word frequency, digit symbol coding and verbal memory upon the treatment with pramipexole. However, no significant correlations between changes in clinical variables including sleep duration, depression, and cognitive functions before and after the treatment. The other study showed that after a three-month treatment with pramipexole in twenty RLS patients, almost all cognitive investigated domains improved. Improvement in these cognitive domains was associated with improvement in RLS score, quality of life, depressive and anxiety symptoms, and daytime sleepiness. However, as they did not show correlation analysis between cognitive improvement and change of RLS severity, daytime sleepiness and depression score, factors that may be associated with cognitive improvement could not be identified.

A recent study reported the effects of pramipexole on the Sternberg working memory task performance evaluated by 
ERP study. ${ }^{22}$ RLS symptoms as well as sleep quality and depression were significantly improved after treatment. Reaction time and omission error was significantly reduced following treatment with pramipexole. Parietal P300 amplitude increased significantly after the treatment (Fig. 2). Increase in frontal P300 amplitude correlated significantly with improvement of sleep quality and RLS symptoms.

In summary, dopamine agonist may improve cognitive performance in RLS. However, it remains unclear whether this is a direct effect on RLS symptoms or an indirect effect of improvement of sleep duration, daytime sleepiness, or depression, or all of them.

\section{Event-Related Potential Study in RLS}

Electroencephalogram (EEG) allows non-invasive moni- toring of brain processes with excellent temporal resolution. EEG during relaxed wakefulness, that is, during the resting state, reflects a particularly important state of arousal, which can be characterized by frequency analysis. ERP is a measured brain response that is direct result of a specific sensory, cognitive, or motor event. ${ }^{23}$ An ERP study provides useful information for exploring cognitive function and non-invasive monitoring of brain processes with excellent temporal resolution and modest spatial resolution covering the whole brain. ERP measurements during tasks may provide more sensitive means of objectively assessing cognitive function. In particular, P300 has been proposed as an index of multiple cognitive processes, including attention, context updating, and processing resource allocation. ${ }^{24}$ Thus, EEG and ERP studies might be useful for exploring cognitive functions in RLS possibly associated with attention and/or arousal state dysfunctions.
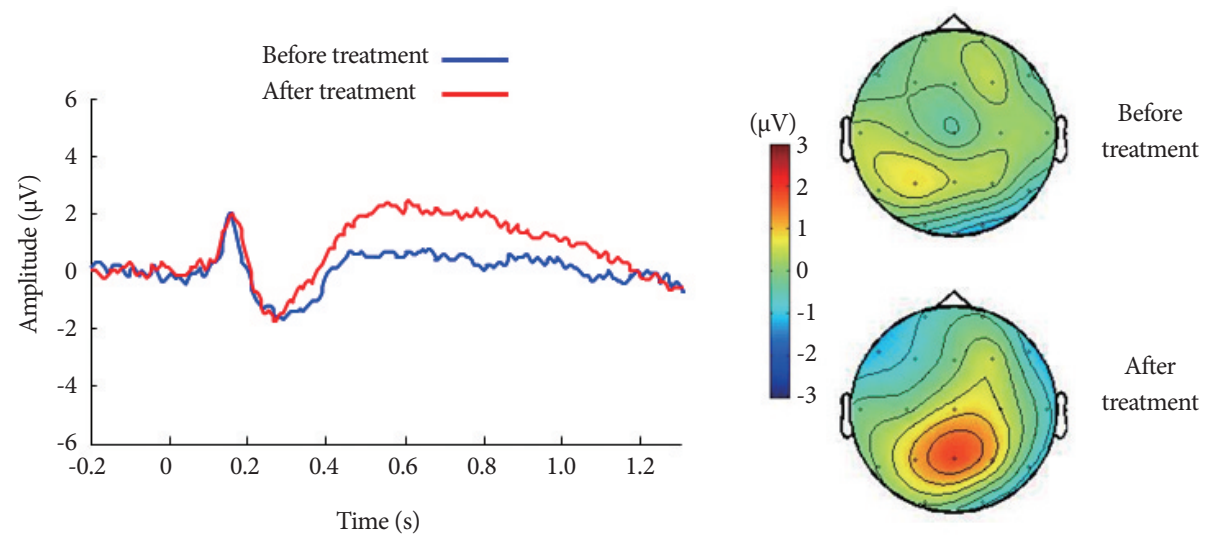

Figure 2. Increased P300 amplitude during Sternberg working memory task following pramipexole administration in patients with restless legs syndrome. Left panel is an event-related potential plot at Pz electrode. Right panel is a topographic distribution of averaged P300 amplitude between $400 \mathrm{~ms}$ and $700 \mathrm{~ms}$. Modified from Kim SM, et al. Sleep Med 2014;15:808-815. ${ }^{29}$
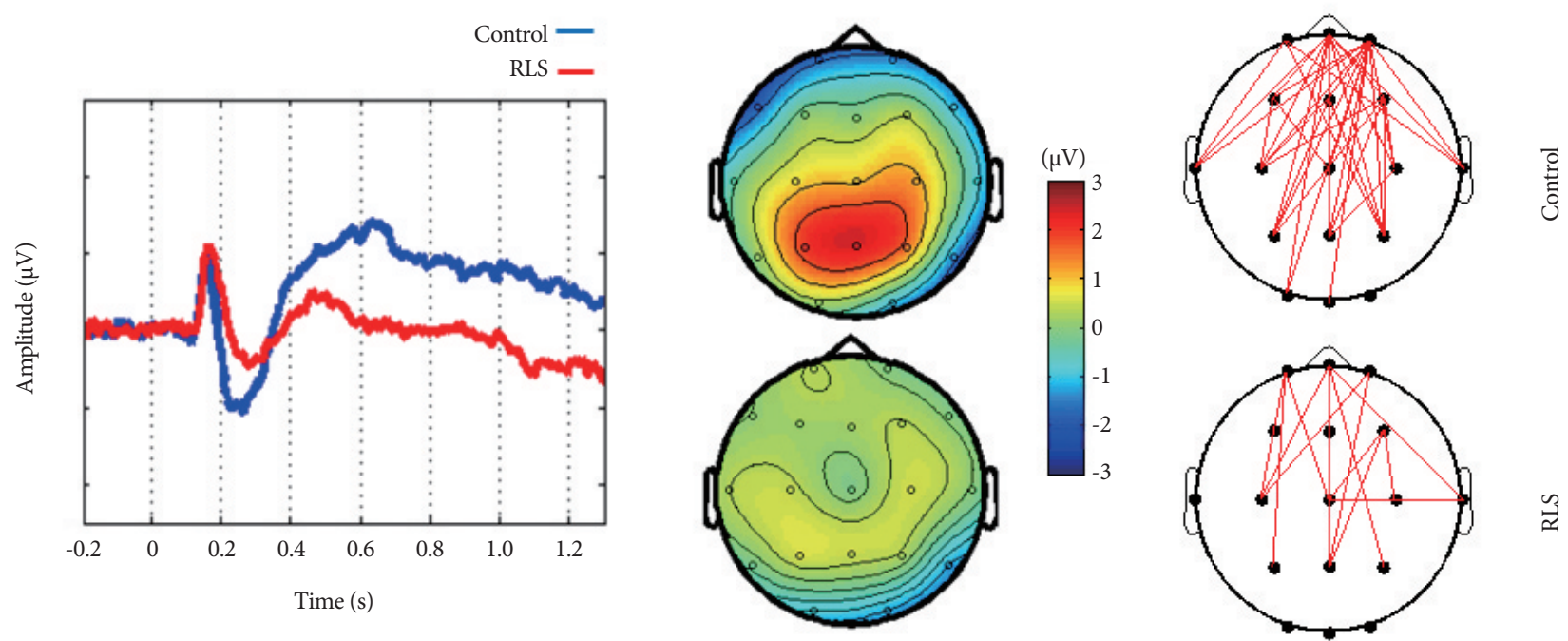

Figure 3. Comparison of event-related potentials (ERPs) during visual oddball paradigm between restless legs syndrome (RLS) and control group. Left panel is an ERP plot at Pz electrode. Middle panel shows topographic distribution of averaged P300 amplitude between $400 \mathrm{~ms}$ and $700 \mathrm{~ms}$. Right panel reveals decreased gamma band phase synchrony, particularly, frontoparietal connection at $450 \mathrm{~ms}$ after stimulus onset. Modified from Choi JW, et al. PLoS One 2012;7:e42312 ${ }^{26}$ and Kim SM, et al. Sleep Med 2014;15:808-815. ${ }^{29}$ 
Jung et al. firstly reported abnormalities in resting EEG and ERP during visual oddball task in 17 patients with RLS compared to 13 age-matched healthy controls. ${ }^{25}$ Wakingresting EEG showed that RLS patients had significantly higher beta activity in frontocentral regions than controls. P300 latency was significantly longer in patients, and patients had significantly lower P300 amplitudes in frontal and central locations. Further analysis revealed that gamma-band activity and phase synchrony, which is known to be associated with working memory, was significantly decreased in RLS patients compared to normal subjects, especially at frontal region. This suggests that cognitive dysfunction in RLS patients may result from less efficient cortical information processing due to the reduced interregional neuronal synchrony as well as alterations in local activity. ${ }^{26}$ These studies support the notion that RLS patients have an underlying cognitive dysfunction.

Working memory deficits might be caused by depression, insomnia, or sleep deprivation, which are common in RLS. ${ }^{27,28}$ Therefore, working memory deficit would be expected in RLS patients. However, most studies, except for one study, ${ }^{7}$ reported that RLS patients have no impairment in either block span backward or digit span backward test. Kim et al. recorded ERP during Sternberg working memory task in severe RLS patients. ${ }^{29}$ The reaction time in RLS patients was significantly longer than controls over all memory load sizes. The P300 amplitude at parietal regions in RLS patients was significantly lower than that in controls regardless of memory load sizes (Fig. 3) and negatively correlated with duration of RLS history in RLS patients.

\section{Conclusions}

Considering that multiple factors are involved in the cognitive process and that different methodologies and subjects were included in various studies, there is no consensus for cognitive impairment in RLS. Cognitive function in RLS seems to be preserved assessed by neurocognitive tests. However, sophisticated methods such as ERP study may help to identify underlying cognitive dysfunction in RLS. Treatment with dopamine agonist significantly improves cognitive performance in RLS. Further studies including functional neuroimaging study are needed to confirm whether cognition in RLS patients is impaired or not.

\section{Acknowledgments}

This research was supported by the National Research Foundation of Korea (NRF) grant funded by the Korean government (MEST) (no. 20110029740) and by future environmental R\&D grant funded by the Korea environmental industry and technology institute (No. RE201206020).

\section{REFERENCES}

1. Fulda S, Beitinger ME, Reppermund S, Winkelmann J, Wetter TC. Short-term attention and verbal fluency is decreased in restless legs syndrome patients. Mov Disord 2010;25:2641-2648.

2. Fulda S, Szesny N, Ising M, et al. Further evidence for executive dysfunction in subjects with RLS from a non-clinical sample. Sleep Med 2011;12:1003-1007.

3. Pearson VE, Allen RP, Dean T, Gamaldo CE, Lesage SR, Earley CJ. Cognitive deficits associated with restless legs syndrome (RLS). Sleep Med 2006;7:25-30.

4. Celle S, Roche F, Kerleroux J, et al. Prevalence and clinical correlates of restless legs syndrome in an elderly French population: the synapse study. J Gerontol A Biol Sci Med Sci 2010;65:167-173.

5. Driver-Dunckley E, Connor D, Hentz J, et al. No evidence for cognitive dysfunction or depression in patients with mild restless legs syndrome. Mov Disord 2009;24:1840-1842.

6. Galbiati A, Marelli S, Giora E, Zucconi M, Oldani A, Ferini-Strambi L. Neurocognitive function in patients with idiopathic Restless Legs Syndrome before and after treatment with dopamine-agonist. Int J Psychophysiol 2015;95:304-309.

7. Lee HB, Ramsey CM, Spira AP, Vachon J, Allen R, Munro CA. Comparison of cognitive functioning among individuals with treated restless legs syndrome (RLS), untreated RLS, and no RLS. J Neuropsychiatry Clin Neurosci 2014;26:87-91.

8. Rist PM, Elbaz A, Dufouil C, Tzourio C, Kurth T. Restless Legs Syndrome and Cognitive Function: A Population-based Cross-Sectional Study. Am J Med 2015 [Epub]. http://dx.doi.org/10.1016/j.amjmed.2015.04.011.

9. Gamaldo CE, Benbrook AR, Allen RP, Oguntimein O, Earley CJ. A further evaluation of the cognitive deficits associated with restless legs syndrome (RLS). Sleep Med 2008;9:500-505.

10. Moon YJ, Song JY, Lee BU, Koo YS, Lee SK, Jung KY. Comparison of Cognitive Function between Patients with Restless Legs Syndrome and Healthy Controls. Sleep Med Res 2014;5:20-24.

11. Allen RP, Picchietti D, Hening WA, et al. Restless legs syndrome: diagnostic criteria, special considerations, and epidemiology. A report from the restless legs syndrome diagnosis and epidemiology workshop at the National Institutes of Health. Sleep Med 2003;4:101-119.

12. Winkelman JW, Redline S, Baldwin CM, Resnick HE, Newman AB, Gottlieb DJ. Polysomnographic and health-related quality of life correlates of restless legs syndrome in the Sleep Heart Health Study. Sleep 2009;32:772-778.

13. Cortese S, Konofal E, Lecendreux M, et al. Restless legs syndrome and attention-deficit/hyperactivity disorder: a review of the literature. Sleep 2005;28:1007-1013.

14. Wagner ML, Walters AS, Fisher BC. Symptoms of attention-deficit/hyperactivity disorder in adults with restless legs syndrome. Sleep 2004; 27:1499-1504.

15. Hornyak M, Kopasz M, Berger M, Riemann D, Voderholzer U. Impact of sleep-related complaints on depressive symptoms in patients with restless legs syndrome. J Clin Psychiatry 2005;66:1139-1145.

16. Sevim S, Dogu O, Kaleagasi H, Aral M, Metin O, Camdeviren H. Correlation of anxiety and depression symptoms in patients with restless legs syndrome: a population based survey. J Neurol Neurosurg Psychiatry 2004;75:226-230.

17. Garcia-Borreguero D, Ferini-Strambi L, Kohnen R, et al. European guidelines on management of restless legs syndrome: report of a joint task force by the European Federation of Neurological Societies, the European Neurological Society and the European Sleep Research Society. Eur J Neurol 2012;19:1385-1396.

18. Manconi M, Ferri R, Zucconi M, et al. Dissociation of periodic leg movements from arousals in restless legs syndrome. Ann Neurol 2012; 71:834-844.

19. Lee HB, Hening WA, Allen RP, et al. Restless legs syndrome is associat- 
ed with DSM-IV major depressive disorder and panic disorder in the community. J Neuropsychiatry Clin Neurosci 2008;20:101-105.

20. Cho YW, Hong SB, Kim do H, et al. The effect of ropinirole on the quality of life in patients with restless legs syndrome in Korea: an 8-week, multicenter, prospective study. J Clin Neurol 2013;9:51-56.

21. Kim M, Song JY, Kim SM, Moon YJ, Koo YS, Jung KY. Effect of Pramipexole on Cognitive Functions in Restless Legs Syndrome. J Korean Sleep Res Soc 2014;11:16-20.

22. Jung KY, Kim SM, Song JY, et al. Sternberg working memory performance following treatment with pramipexole in patients with moderate-to-severe restless legs syndrome. Sleep Med 2015;16:703-708.

23. Jung KY, Cho JW, Joo EY, et al. Cognitive effects of topiramate revealed by standardised low-resolution brain electromagnetic tomography (sLORETA) of event-related potentials. Clin Neurophysiol 2010;121: 1494-1501.

24. Polich J. Updating P300: an integrative theory of P3a and P3b. Clin
Neurophysiol 2007;118:2128-2148.

25. Jung KY, Koo YS, Kim BJ, et al. Electrophysiologic disturbances during daytime in patients with restless legs syndrome: further evidence of cognitive dysfunction? Sleep Med 2011;12:416-421.

26. Choi JW, Ko D, Lee GT, Jung KY, Kim KH. Reduced neural synchrony in patients with restless legs syndrome during a visual oddball task. PLoS One 2012;7:e42312.

27. Baddeley A. Working Memory and Emotion: Ruminations on a Theory of Depression. Rev Gen Psychol 2013;17:20-27.

28. Lovato N, Lack L, Wright H, Cant M, Humphreys J. Working memory performance of older adults with insomnia. J Sleep Res 2013;22:251257.

29. Kim SM, Choi JW, Lee C, et al. Working memory deficit in patients with restless legs syndrome: an event-related potential study. Sleep Med 2014;15:808-815. 